\title{
Inminente Hibernación Solar 2020
}

Maribel S. Guerrero Vásquez

\section{Resumen}

El Ciclo solar $23^{\circ}$, es el vigésimo tercer ciclo, registrado cuidadosamente desde que iniciaron las observaciones de manchas solares en occidente, en el siglo XVII. En este trabajo se pretende demostrar estadísticamente, la tendencia hacia una hibernación solar, donde pueden ser muchos años en que el Sol no produzca ninguna mancha solar, en este caso específico se estudió la actividad solar final del ciclo 23 y comienzos del ciclo 24. Planteando la tendencia de baja actividad en el Sol y que continuará por varios años, como se ha visto que sucedieron, en los cinco periodos, con espacios aproximados a 200 años o menos cada uno, diversos períodos excepcionales de baja actividad solar durante el último milenio. Estos períodos son: el de Norman (1010) el de Wolf (hacia el año 1300), Spoerer (1500), Maunder (1645 - 1715) y Dalton (1800-1830). Por lo que no resultaría extraño otro acontecimiento característico similar, en un futuro cercano; porque han transcurrido 186 años a la fecha, desde el último mínimo, el de Dalton desde el año 1800 a 1830. Se ha observado que la actividad solar, volvió a descender desde el año 2011 y ocurrió un pequeño rebrote en 2013. Así una buena parte de la comunidad científica espera que cuando finalice este ciclo 24 de manchas solares, es muy posible que el astro entre en hibernación. De seguir con la tendencia a la baja actividad solar, consecuentemente puede bajar la temperatura en nuestro planeta Tierra, como se ha visto en el pasado y arrojada por los resultados estadísticos de esta investigación.

Palabras Clave: manchas solares, actividad solar, ciclo solar, tormentas geomagnéticas, Sol.

\section{Abstract}

The solar cycle $23^{\circ}$, is the twenty-third cycle began carefully recorded since observations of sunspots in the West, in the seventeenth century. This paper aims to demonstrate statistically, the trend toward a solar hibernation, they can be many years in the Sun no Sunspot does not occur in this case specific study final solar 
activity cycle 23 and the beginning of the 24th cycle The raising trend low activity in the sun and will continue for several years, as we have seen that occurred in five periods, with an approximate 200 years or less, different exceptional periods of low solar activity during the last millennium. These periods are: Norman (1010), the Wolf (1300), Spoerer (1500), Maunder (1645-1715) and Dalton (1800-1830). So it does not be strange another similar characteristic event in the near future. Because 186 years have elapsed date from the last minimum, Dalton from the year 1800 to 1830. It has been observed that solar activity, became a descender from the year 2011 and occurred small outbreak in 2013. So much of the scientific community expects that when complete this cycle 24 sunspot, it is very possible may start the star can enter a period of hibernation. To continue the trend to low solar activity, therefore it can lower the temperature on our planet Earth, as we have seen in the past and thrown by the statistical results in this research.

Keywords: sunspots, solar activity, solar cycle, geomagnetic storms, Sun.

Maribel S. Guerrero Vásquez. (marsuy3000@gmail.com).Facultad de Ciencias Espaciales Departamento de Astronomía y Astrofísica. Universidad Nacional Autónoma de Honduras.

Fecha de Recepción: 9 de agosto de 2016, fecha de aprobación:11 de octubre de 2016. 


\section{INTRODUCCIŐN}

La presente investigación consiste en encontrar las anomalías del ciclo de mínima actividad solar número 24 , porque reflejan la prolongación de ese ciclo al presente. La metodología planeada inicia estudiando primero las manchas solares desde el final del ciclo 23, e inicio del ciclo 24 al 2014. Seguidamente en un estudio estadístico de manchas solares algunos fenómenos dinámicos como las Tormentas Geomagnéticas. Y los tipos de polaridad reflejada como indicadores de la mínima actividad en ese periodo de tiempo. Con los datos obtenidos en los sitios disponibles como el sumario de las regiones solares activas, del NOAA de Hawái, entre otros. En diferentes longitudes de onda o frecuencia de la radiación electromagnética. Se presume que posiblemente se fortalecerá la hipótesis propuesta: "La actividad del actual ciclo solar 24 y el siguiente continuarán en descenso y consecuentemente también la Temperatura en la Tierra". El Sol sobre el horizonte en el planeta Tierra.

\section{METODOLOGÍA}

Para concretar la investigación se buscaron los datos apropiados, para seleccionar y archivarlos, esta información digital finalmente fue obtenida de la página web del MEES Solar Observatory, uno de los observatorios de la Universidad de Hawái Haleakala. Localizados en Maui, Hawái, con una elevación de 3,054 m.s.n.m. aproximadamente. Se utilizaron datos fotosféricos de manchas solares y tormentas geomagnéticas, que coincidieron en fecha con esa actividad solar, del fin del ciclo 23 en diciembre 2007, e inicios del ciclo 24 en enero 2008, hasta el año 2014. Después de encontrar y seleccionar la información se ordenó para hacer los cálculos necesarios y finalizar con las estadísticas, correspondientes a los años de estudio. Finalmente se resumieron tabulados los resultados cuantitativos, graficar y hacer un análisis, de acuerdo a la hipótesis propuesta en la investigación.

\section{RESULTADOS}

El clico 23 terminó y comenzó el ciclo 24 oficialmente el 4 de enero del 2008, inicio su máximo, con baja actividad, como continuación del mínimo anterior hasta enero 2010 donde se observaron menos de diez manchas y volvió a decaer mucho más a finales del 2010 y comienzos del 2011. 
Los meses de febrero 2012, octubre 2013 y agosto 2014 resultaron con cero manchas (Hathaway David 2012) del Centro Marshall para Vuelos Espaciales, NASA). Se puede observar que este ciclo alcanzó la cantidad máxima de 20 manchas, en uno de sus picos superiores, de todos los datos encontrados hasta abril del 2016. En el año 2015 hubo un pequeño rebrote de manchas.

Tabla 1. Cantidad anual de manchas 2010 al 2016

\begin{tabular}{|r|r|}
\hline Año & Cantidad anual de manchas \\
\hline 2010 & 66 \\
\hline 2011 & 136 \\
\hline 2012 & 133 \\
\hline 2013 & 103 \\
\hline 2014 & 71 \\
\hline 2015 & 21 \\
\hline 2016 & 5 \\
\hline & \\
\hline
\end{tabular}

La Tabla 1 muestra el resumen de datos por año. A partir de la cual se han graficado estos datos de manchas solares, correspondientes a enero 2010 hasta abril 2016, la tendencia es bien clara, hacia cero. Los datos resultantes de esta investigación evidencian en la Tabla 1, que los ciclos no tienen la forma de campana de los ciclos anteriores, con una distribución normal, sino que hay muchos picos altos y bajos. Por lo que el promedio de esa curva evidencia lo que podemos esperar en cuanto a la aparición o no de manchas solares. 


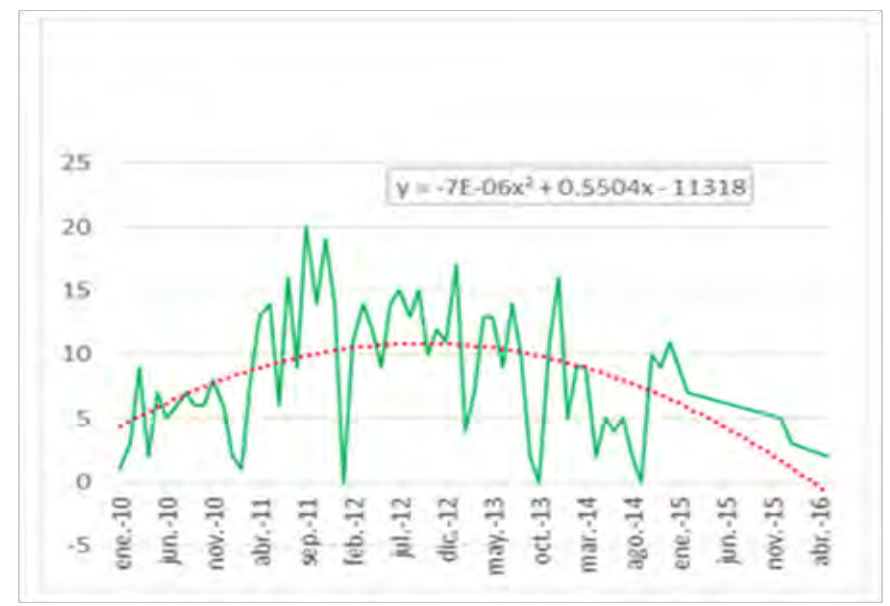

Figura 1. Manchas Solares 2010- 2016 y la tendencia.

El gráfico superior muestra la cantidad de manchas mensuales por año, desde 2010 al 2016 (curva verde). La línea de tendencia logarítmica (en rojo) y la ecuación de la curva. Fuente imagen M. Guerrero 2016

Estudiando la curva de tendencia polinomial, vemos que los siguientes períodos están basados en los datos de eventos pasados desde enero del 2010 hasta los datos recientes de abril 2016. La tendencia nos dice que posiblemente los siguientes ciclos máximos también serán atípicos, con pocas o ninguna mancha solar, con esta predicción estadistica. 
Tabla 2. Picos inferiores, cantidad de manchas igual a cero.

\begin{tabular}{|l|l|}
\hline Rango de tiempo donde se observaron picos & Tiempo entre picos \\
inferiores en la cantidad de manchas & bajos \\
\hline Enero 2010 - Dic 2010 & 12 meses \\
\hline Diciembre 2010 - Febrero 2012 & 14 meses \\
\hline Febrero 2012 - Diciembre 2012 & 11 meses \\
\hline Diciembre 2012- Octubre 2013 & 10 meses \\
\hline Octubre 2013 - Agosto 2014 & 10 meses \\
\hline Tiempo promedio & 11.4 meses \\
\hline
\end{tabular}

La tabla anterior resume la desaparición total de manchas solares, representados en los picos inferiores del gráfico de la Figura 1, desde enero 2010 hasta abril 2016.

En seis años de máxima (oficial) baja (real) actividad solar, hubo en promedio cada 11 meses; al menos un día sin ninguna mancha en el Sol. Se puede prever el comportamiento del ciclo de mínima actividad cercana para el año 2020, que bien puede cerrar otro ciclo de periodos más fríos sobre la Tierra, similar a los acontecidos aproximadamente en 200 años cada uno, sin ninguna mancha y bajas temperaturas, similares a los mínimos históricos arriba mencionados. La NASA tiene algunos datos interesantes que indican que la actividad solar está relacionada con el clima, (Pesnell Dean 2011) científico del Observatorio Dinámico Solar (SDO) de la NASA. 
La siguiente Figura 2 muestra la gráfica del comportamiento de las tormentas geomagnéticas, otro indicador de la actividad del Sol y la tendencia de la curva en negro hasta 2012. (Guerrero V. Maribel 2012), de seguir bajando, en concordancia con las manchas solares, en una parte amplia del periodo estudiado actualmente. En los cinco periodos históricos de mínima actividad se observaron auroras boreales, o sea que hubo otros eventos solares, dinámicos, pero que no influyeron en las bajas temperaturas registradas.

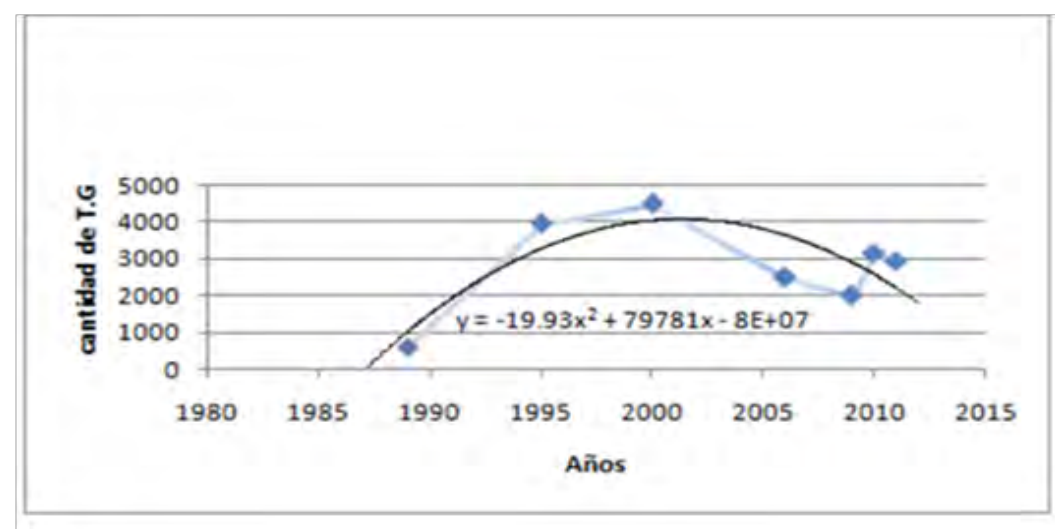

Figura 2. Cantidad de tormentas geomagnéticas (TG) desde 1989 al presente septiembre 2011 y la curva de tendencia polinomial.

Curva del comportamiento de la actividad solar, en el acontecimiento de tormentas geomagnéticas ( $T: G)$ y tendencia de perturbaciones del campo magnético de años anteriores y la ecuación de la curva. Fuente M. Guerrero 2012, El Mito del Calentamiento Global.

Con esta tendencia se presume que continuara el descenso de actividad solar en todos los fenómenos atmosféricos del Sol, hasta que lleguemos a un nuevo periodo de bajas temperaturas globales a corto plazo. 


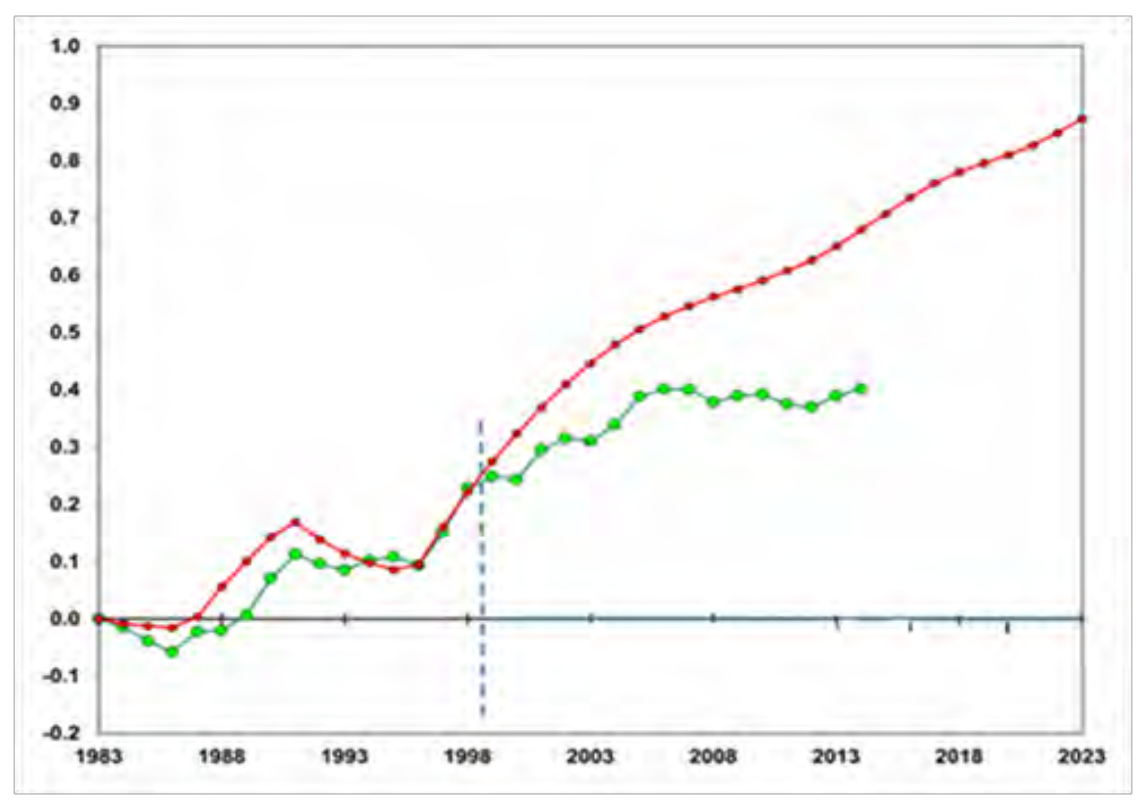

Figura 3. Modelos climáticos VS. Observaciones de Temperatura promedio $\left({ }^{\circ} \mathrm{C}\right)$ de la superficie

El gráfico muestra la curva roja de modelos climáticos promedio y la curva verde de observaciones de temperatura superficial. El eje vertical representa la temperatura en grados centígrados, el eje horizontal representa los años desde 1983 hasta 2023. Fuente //drroyspencer.com, editado por M. Guerrero 2012.

En las curvas del grafico anterior, se puede observar la temperatura media de 1983 a 1995 entre la observada y la modelada. Mientras que los primeros años del acuerdo entre los modelos climáticos y observaciones, el uso de modeladores obligó a creer que su sensibilidad climática, asumida sobre todo en el CO2 era correcta. 1997-2013 una pausa en el calentamiento que sugiere que no se descartan fuentes de calentamiento o enfriamiento natural. Como ha ocurrido en el pasado. Se ve que de 1998 al 2023, la sensibilidad del modelo utilizado fue demasiado alta para compensar la diferencia y ahora los modelos escapan de la realidad porque son muy sensibles y así las predicciones aumentan excesivamente el calentamiento, completamente fuera de la realidad, como se ve en la curva roja de la Figura 4. Mientras que la temperatura observada al presente, en el máximo solamente aumento 0.2 grados centígrados. 


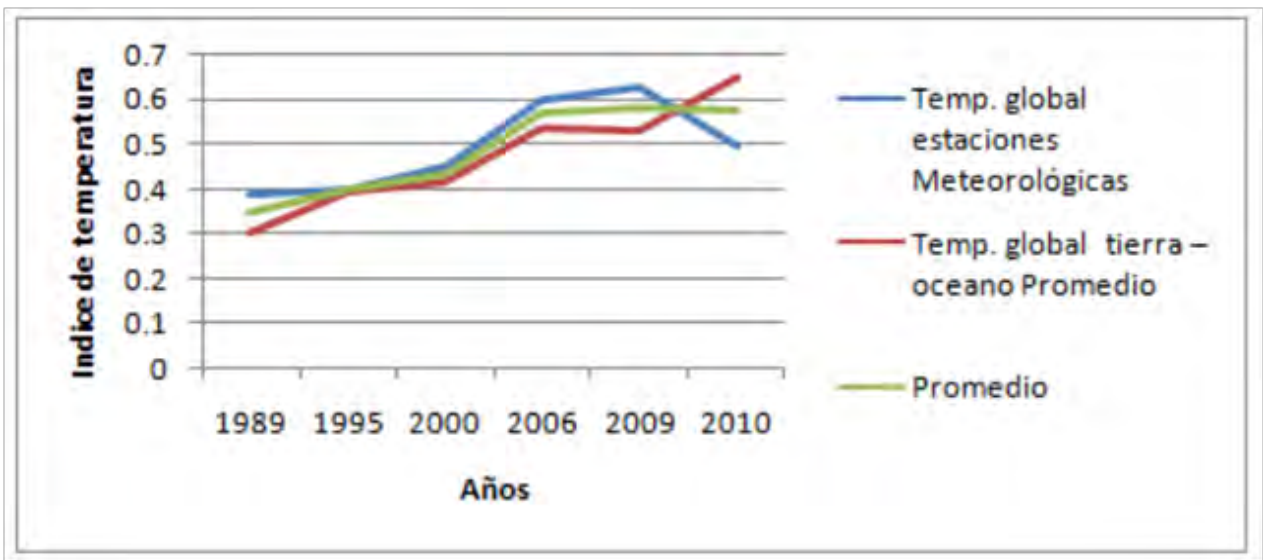

Figura 4. Índices de temperatura global estaciones meteorológicas, global tierra - océano promedio y promedio de ambas.

El gráfico muestra los índices de temperatura Tierra - océano en color azul y estaciones meteorológicas color rojo, desde 1989 al 2010, el promedio en color verde. Fuente M. Guerrero 2012, El Mito del Calentamiento Global

Por otra parte, se ha encontrado por métodos cuantitativos, similitud entre el comportamiento de la actividad solar y el índice de temperatura global promedio (Figura 4) donde las curvas muestran la posibilidad de correlacionar el enfriamiento o calentamiento global de la Tierra, como parte de un ciclo natural. (Guerrero V. Maribel 2012).Y la temperatura promedio se mantuvo constante y ha seguido el mismo comportamiento como se ve en la Figura 4.

Para el ciclo 23 la cantidad máxima fue de 80 manchas y para el ciclo 24 la cantidad máxima no ha pasado de 35 manchas, con varios días sin ninguna macha, ambos datos para los máximos solares. Por eso se le ha llamado en este trabajo máxima actividad anulada (MAA).

En los ciclos de mínima actividad el ciclo 23 ha habido muchos días sin ninguna mancha y aun no se inicia el ciclo de mínima actividad 24, que se prevé según las estadísticas hechas que posiblemente no haya manchas en el disco solar durante ese tiempo. 


\section{DISCUSIÓN}

A pesar que el máximo solar del ciclo 23 fue superior en cantidad al ciclo máximo actividad 24, que aún no termina. Estadísticamente se ve la tendencia hacia cero manchas, por tiempo más prolongado, ocasionalmente habrá más días sin ninguna mancha. Ya que para el inicio del ciclo 25 , posiblemente se verán cambios severos como el descenso de la temperatura superficial de nuestro planeta, de continuar la baja actividad solar o sea la escases de manchas solares.

\section{CONCLUSIONES}

El ciclo anterior 23 presentó dos picos muy altos en el máximo. Y el mínimo fue más prolongado que el del ciclo 22. Vemos que el actual ciclo 24 también presento dos picos, pero mucho más bajos, respecto a la cantidad de manchas del ciclo 23. Los resultados de esta investigación confirman la tendencia hacia cero manchas en el cercano ciclo 25.

En la actualidad somos testigos de un periodo de prolongada y débil actividad del Sol, los máximos han sido realmente imperceptibles y los mínimos con pocas 0 ninguna mancha solar (mínimos profundos).

Pero de las curvas de tendencia se puede predecir que el Sol va bajando su actividad y que este ciclo MAA, terminará unido a la baja actividad del inicio del próximo ciclo 25; similar al final del ciclo 23 e inicio del 24. Por otra parte, el comportamiento de la temperatura, analizada, también coincide con el comportamiento atípico mostrado por las tormentas geomagnéticas, toda la radiación solar nos llega en forma de luz y calor, debido a la energía cinética (energía asociada a los cambios de velocidad). Estas similitudes en ciclos de actividad solar mínima, prolongados puede contribuir a alcanzar muy bajas temperaturas sobre la Tierra, en un futuro cercano. Se puede prever la similitud, entre el comportamiento estadístico del ciclo de máxima actividada nulada (MAA) y el inicio del siguiente ciclo antes del año 2020, tiempo en el que la Tierra probablemente descenderá mucho su temperatura superficial. Además se suma otra variable al cambio climático y a la temperatura superficial de nuestro planeta, las corrientes oceánicas de convección, que según estudios recientes se han reducido; aun así se ha observado que la temperatura de la Tierra varía constantemente y de manera más drástica con la variación de la actividad solar (T. Ramírez Jenine 2006). De acuerdo a esta investigación se puede 
correlacionar la baja actividad solar actual, con las temperaturas casi constantes reales, observadas en el periodo de estudio y de cumplirse la tendencia de baja actividad solar teórica, tendríamos en el umbral otro enfriamiento histórico del planeta Tierra.

\section{BIBLIOGRAFÍA}

- Are the sunspots really vanishing? Anomalies in solar cycle 23 and implications for long- term models and proxies. Clette, Frédéric; Lefèvre, Laure.

- Declining solar activity in solar cycles 22 and 23 and their inner heliospheric signatures Padmanabhan, Janardhan(abstrac); Ananthakrishnan, Subramaniam; Bisoi, SusantaKumar.

- Empirical Relationship Between CME Parameters and Geo-effectiveness of Halo CMEs in the Rising Phase of Solar Cycle 24 (2011 - 2013).

- Geomagnetic Activity Chart, disponible en http://hirweb.nict.go.jp/sedoss/ geoact.

- Gosling, J.T, 1999, Journal of geophysical research, Volumen 104, p. 12515 a 12524.

- Gopalswamy, N.; Yashiro, S.; Xie, H.; Akiyama, S.; Mäkelä Hathaway, D. $\mathrm{H}$. Flux Transport and the Sun's Global Magnetic Field (Invited), American Geophysical Union, Fall Meeting 2010, abstract \#SH41D-01.

- Howard, R. A. et al, 1985, Journal of geophysical research, Vol. 90. Recuperado de: $\quad$ http://www.docstoc.com/docs/58393503/An-Historical-Perspective-toCME.

- Impact on Earth and Planets, Proceedings of the International Astronomical Union, IAU Symposium, Volume 264, p. 202-209.

- Journal of Space Weather and Space Climate, Volume 2, id.A06.

- Major Eruptive Active Region Based on SDO/HMI Observation. The Astrophysical Journal, Volume 748, Issue 2, article id. 77, 15 pp. (2012). (ApJ Homepage). 
- Prediction of solar activity cycles by assimilating sunspot data into a dynamo model. Solar and Stellar Variability Kitiashvili, Irina N.;Kosovichev, Alexander G.

- Solar Active Region Classification and Flare Forecasting American Geophysical Union, Fall Meeting 2010, abstract \#SM54A-07.

- Sun, Xudong; Hoeksema, J. Todd; Liu, Yang; Wiegelmann, Thomas; Hayashi, Keiji; Chen, Qingrong; Thalmann, Julia, Evolution of Magnetic Field and Energy in a The Peculiar Behavior of Halo Coronal Mass Ejections in Solar Cycle 24Gopalswamy, N.; Xie, H.; Akiyama, S.; Mäkelä, P.; Yashiro, S.; Michalek, G.

- 39th COSPAR Scientific Assembly. Held 14-22 July 2012, in Mysore, India. Abstract. 